\title{
Shock séptico, hipercalcemia y síndrome hemofagocítico por paracoccidioidomicosis en una niña de 3 años
}

\section{Septic shock, hypercalcemia and hemophagocytic syndrome due to paracoccidioidomycosis in a 3-year-old girl}

\section{RESUMEN}

Objetivo: Reportar el caso de una niña que presentó shock séptico, hipercalcemia y síndrome hemofagocítico por paracoccidioidomicosis. Reporte de caso: Paciente mujer de 3 años con 2 meses de enfermedad caracterizada por fiebre, adenopatías, pérdida de peso, distensión abdominal y anemia severa. Llegó en mal estado general, desarrolló shock séptico y un síndrome hemofagocítico. Llamaba la atención la hipercalcemia y lesiones dérmicas. Se le realizó una biopsia de ganglio cervical y aspirado de médula ósea. En esta última se evidenciaron imágenes compatibles con Paracoccidioides, que fueron confirmadas con la tinción de Grocott del ganglio. Se trató con anfotericina B y trimetoprim-sulfametoxazol. La evolución fue favorable y el calcio sérico se mantuvo en rangos normales. Conclusión: Las complicaciones presentadas por paracoccidioidomicosis son raras; sin embargo, el antecedente epidemiológico y el aspirado de médula ósea pueden orientar el diagnóstico y permitir un tratamiento oportuno.

Palabras Clave: Paracoccidioidomicosis; Choque Séptico; Hipercalcemia; Linfohistiocitosis Hemofagocítica; Preescolar (Fuente: DeCS-BIREME).

\section{ABSTRACT}

Objetive: Report the case of a girl who presented septic shock, hypercalcemia and hemophagocytic syndrome due to paracoccidioidomycosis. Case report: 3 -year-old female patient with 2-month illness characterized by fever, lymphadenopathy, weight loss, abdominal distention, and severe anemia. She arrived in poor general condition, developed septic shock and a hemophagocytic syndrome. The hypercalcemia and dermal lesions were striking. A cervical ganglion biopsy and bone marrow aspirate were performed. In the latter, images compatible with Paracoccidioides were found, which were then confirmed with Grocott's stain. She was treated with amphotericin B and trimethoprim-sulfamethoxazole. The evolution was favorable and serum calcium came back to normal ranges. Conclusion: Complications caused by paracoccidioidomycosis are rare; however, the epidemiological history and bone marrow aspirate should guide the diagnosis and allow prompt treatment.

Keywords: Paracoccidioidomycosis; Shock, Septic; Hypercalcemia; Lymphohistiocytosis, Hemophagocytic; Child, Preschool. (Source: DeCS-BIREME).
FILIACIÓN

1. Clínica San Felipe, Lima, Perú.

2. Departamento de Educación Médica, Nicklaus Children's Hospital, Miami, FL, EEUU.

3. Instituto de Patología y Biología Molecular Arias Stella, Lima, Perú.

a. Médico pediatra intensivista.

b. Médica cirujana.

c. Médica hematóloga pediatra.

d. Médico patólogo.

ORCID

1. Ricardo Rodríguez 0000-0002-5905-1297

2. Angela Parra del Riego Freundt-Thurne 0000-0002-9707-9135

3. Carmen Carolina Tokumura Tokumura 0000-0003-1639-9733

4. Ninoska Julia Rojas Soto 0000-0003-0303-1024

5. Jorge Valdés Gómez 0000-0001-6019-9005

\section{CORRESPONDENCIA}

Ricardo Rodríguez Portilla.

Dirección: Distrito de Jesús María. Lima, Perú.

EMAIL

ricardo.rodriguez@upch.pe

\section{CONFLICTOS DE INTERÉS}

Los autores niegan conflictos de interés.

\section{FINANCIAMIENTO}

Autofinanciamiento.

\section{REVISIÓN DE PARES}

Recibido: 24/06/2021

Aceptado: 01/09/2021

\section{COMO CITAR}

Rodríguez-Portilla, R., Freundt-Thurne, A., Tokumura-Tokumura, C. Rojas-Soto, N., \& Valdés-Gómez, J. Shock séptico, hipercalcemia y sindrome hemofagocítico por paracoccidioidomicosis en una niña de 3 años. Revista Del Cuerpo Médico Hospital Nacional Almanzor Aguinaga Asenjo, 2021, 14(3). https://doi.org/10.35434/rcmhnaaa.2021.143.1259

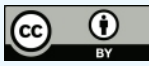

Esta obra está bajo una Licencia Creative Commons Atribución 4.0 Internacional.

Versión Impresa: ISSN: 2225-5109

Versión Electrónica: ISSN: 2227-4731

Cross Ref. DOI: 10.35434/rcmhnaa

OJS: https://cmhnaaa.org.pe/ojs 


\section{INTRODUCCIÓN}

La paracoccidioidomicosis en niños es una micosis sistémica potencialmente mortal que requiere un alto grado de sospecha diagnóstica ${ }^{(1)}$. Es causada por un hongo dimórfico llamado Paracoccidioides brasiliensis y es considerada una infección endémica en países de América Latina con clima tropical $^{(2)}$. En Perú, la tasa anual media de casos llega a 3 , mientras que en Brasil a 579 , y su letalidad alcanza un 3 a $5 \%$.

Esta infección se presenta de dos formas. La aguda o subaguda, característica de niños y jóvenes, quienes desarrollan linfadenopatías generalizadas, lesiones mucocutáneas y compromiso visceral. Mientras que en adultos predomina la forma crónica, que afecta principalmente pulmones, vía área superior y mucosas de boca y nariz ${ }^{(4)}$.

Las complicaciones más comunes, aunque infrecuentes, son la insuficiencia renal y disturbios electrolíticos ${ }^{(5)}$. Complicaciones como shock séptico, hipercalcemia o síndrome hemofagocítico han sido descritas pocas veces en la literatura ${ }^{(5)}$ y según nuestra revisión no se ha encontrado un reporte que presente estas tres complicaciones juntas en un mismo paciente. Por ello, relatamos el caso de una niña con paracoccidioidomicosis diseminada que desarrolló shock séptico, hipercalcemia y síndrome hemofagocítico.

\section{REPORTE DE CASO}

Paciente mujer de 3 años, natural y procedente de la Provincia de Chanchamayo en el Departamento de Junín en la selva central de Perú. No tenía antecedentes de importancia, presentaba vacunas completas, y un índice de masa corporal en el percentil 18 hasta antes del inicio de los síntomas. Ingresó referida del Hospital de su localidad con diagnóstico de síndrome linfoproliferativo.

Inició su enfermedad dos meses y medio antes de ingresar a la Clínica con fiebre de frecuencia semanal. Un mes después se agregó distensión abdominal progresiva asociada a dolor, por lo que acudió a consulta en donde evidenciaron hepatoesplenomegalia y crecimiento ganglionar en axila derecha. Además, la fiebre se tornó más frecuente apareciendo cada tres días. Tres semanas antes del ingreso, notaron adenomegalias cervicales, hiporexia, adinamia, ictericia y fiebre interdiaria por lo que fue internada en el Hospital Regional de su localidad con el diagnóstico de probable linfoma. Una vez hospitalizada aparecieron múltiples lesiones papulares de base eritematosa, centro umbilicado y algunas costrosas en cara y tórax anterior;

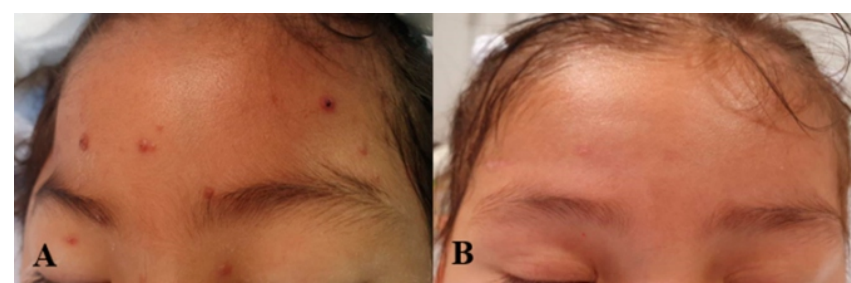

Figura 1

Evolución de lesiones dérmicas papulares de base eritematosa y centro umbilicado, algunas con superficie costrosa. (A) Al ingreso. (B) Al alta. además, fue transfundida por una anemia severa de $5.5 \mathrm{~g} / \mathrm{dl}$. Evolucionó con deterioro clínico, por lo que fue referida a una institución con mayor capacidad resolutiva.

Al examen físico, el día de la admisión, la paciente lucía adelgazada, en mal estado general, e ictérica. Además, se encontraba febril, taquicárdica, taquipnéica, persistía con las lesiones dérmicas ya descritas (Figura 1A), adenomegalias axilares y cervicales (Figura 2A) y hepatoesplenomegalia. Los exámenes auxiliares evidenciaron leucocitosis en $20300 / \mu l$, desviación izquierda con abastonados en $1624 / \mu \mathrm{l}$, eosinofilia en $812 / \mu \mathrm{l}$ y anemia en $8,5 \mathrm{~g} / \mathrm{dl}$, la cual se mantenía baja pese a ser transfundida en 2 oportunidades. Llamaba la atención la hipercalcemia en 15,2 mg/dl; además, de hipoalbuminemia de $1,9 \mathrm{~g} / \mathrm{dl}$ e hipergammaglobulinemia en $6,3 \mathrm{~g} / \mathrm{dl}$. Como parte del estudio diagnóstico se realizó una biopsia de ganglio cervical y un aspirado de médula ósea (AMO). Este último evidenció imágenes compatibles con Paracoccidioides (Figura 3) y la citometría de flujo fue negativa para células malignas.

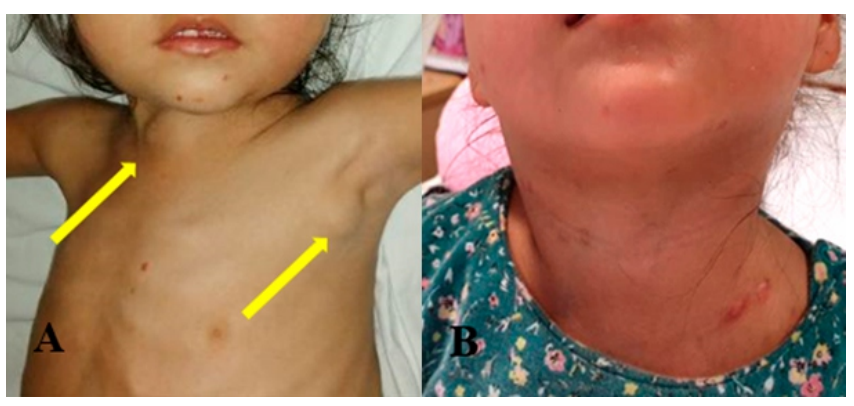

Figura 2.

Evolución de linfadenopatías. (A) Al ingreso. (B) Al alta.

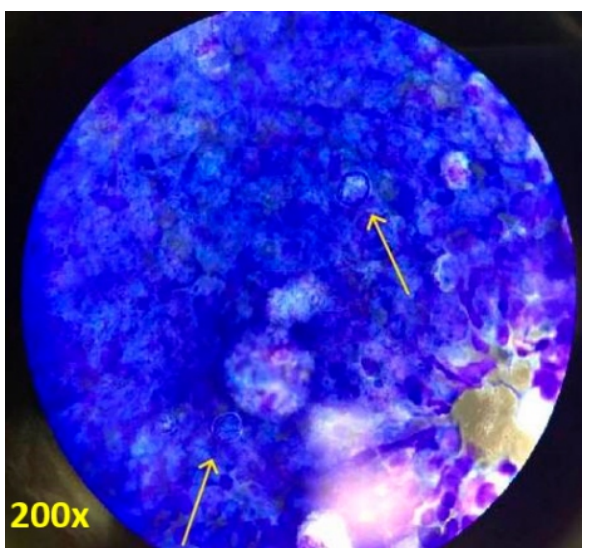

Figura 3.

Aspirado de médula ósea con tinción de Wright. Las flechas señalan estructuras compatibles con Paracoccidioides.

Durante la hospitalización, inició tratamiento con ceftriaxona y se le agregó anfotericina $B$ luego del resultado del AMO. Cuatro días después de su ingreso desarrolló shock séptico con insuficiencia respiratoria, requiriendo soporte con ventilación mecánica invasiva y vasoactivos. Por persistir febril, se rotaron antibióticos a meropenem y vancomicina. Además, por residuo gástrico bilioso y ayuno prolongado inició nutrición parenteral. El calcio llegó a valores de hasta 
Revista del Cuerpo Médico del HNAAA, Vol 14 (3) - 2021

$17,4 \mathrm{mg} / \mathrm{dl}$ y fue tratado con suero fisiológico y furosemida. Luego de 8 días el calcio disminuyó a rangos normales, permaneciendo así hasta el alta.

Se evidenció hiperferritinemia de $1156 \mathrm{ng} / \mathrm{ml}$ que llegó hasta $2733 \mathrm{ng} / \mathrm{ml}$, hipertrigliceridemia de $224 \mathrm{mg} / \mathrm{dl}$, hepatoesplenomegalia, fiebre y hemofagocitosis en el aspirado de médula ósea; por lo que se diagnosticó un síndrome hemofagocítico secundario e inició tratamiento con corticoides e inmunoglobulina a $2 \mathrm{~g} / \mathrm{kg}$. También presentó ascitis a tensión que llegó a dificultar la mecánica ventilatoria del respirador, requiriendo paracentesis evacuatorias hasta en 3 oportunidades. 18 días luego del ingreso, la paciente persistía febril pese al cambio de dispositivos invasivos, hemodinámicamente inestable y con reactantes de fase aguda elevados, motivo por el cual se decidió rotar antibióticos a colistina y linezolid, además se administró una segunda dosis de inmunoglobulina. El informe de la biopsia de ganglio concluyó remplazo subtotal del parénquima por proceso inflamatorio crónico granulomatoso con áreas de necrosis, numerosas células gigantes e incontables estructuras fúngicas, que, por su tamaño, forma y presentar gemación múltiple, resultan consistentes con Paracoccidioides (Figura 4), decidiéndose asociar trimetoprim-sulfametoxazol a la anfotericina B.

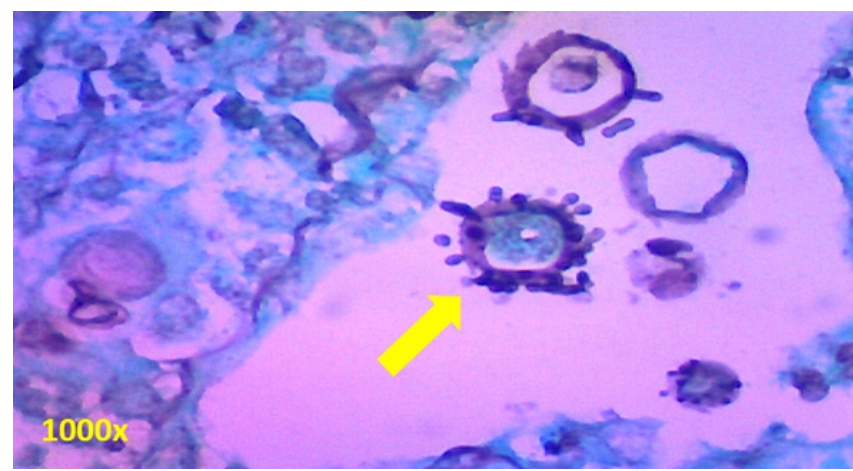

Figura 4.

Biopsia de ganglio con tinción de Grocott. Se observa el aspecto característico en timón de barco del Paracoccidioides.

Posteriormente la evolución fue favorable, 26 días luego del ingreso se consiguió retirar con éxito la ventilación mecánica invasiva y a los 33 días se suspendió la nutrición parenteral. Completó 28 días de trimetoprim-sulfametoxazol y 35 de anfotericina B para luego iniciar itraconazol. Se evidenció mejoría de las lesiones de piel (Figura 1B) y adenomegalias (Figura 2B), además de adecuada ganancia ponderal hasta su basal. Durante su hospitalización, se le realizaron 14 hemocultivos, 4 urocultivos, 4 cultivos de secreción traqueal, 2 cultivos de líquido ascítico, 2 cultivos de Ziehl Neelsen y 1 estudio de quantiferon - TB gold plus, siendo todos los resultados negativos. Con recuperación clínica y laboratorial completa, 81 días después del ingreso la paciente fue dada de alta.

\section{DISCUSIÓN}

La asociación entre síndrome hemofagocítico, hipercalcemia y shock séptico por paracoccidioidomicosis son complicaciones raramente vistas y reportadas en la literatura. Una cohorte de Brasil con 141 niños con paracoccidioidomicosis en un período de 38 años observó que $10(7,6 \%)$ presentaron disturbios electrolíticos, $6(4,5 \%)$ desarrollaron shock séptico y no se reportaron casos de síndrome hemofagocítico ${ }^{(5)}$; haciendo relevante el reporte presentado, debido a la baja frecuencia de estas complicaciones y más rara aún su presentación en un mismo paciente.

La clínica más frecuente en esta patología es inespecífica, donde predominan las linfadenopatías generalizadas, hepatoesplenomegalia, fiebre, baja de peso y palidez, por lo que muchas veces el diagnóstico puede ser tardío y confundido con un síndrome linfoproliferativo; como ocurrió con nuestra paciente que desarrolló complicaciones severas. La primera línea de tratamiento en esta enfermedad, anfotericina $B$, no fue suficiente en este caso y se tuvo que agregar trimetoprim-sulfametoxazol, como se ha sugerido en casos graves ${ }^{(6)}$. Al inicio se sospechó que el estado crítico de la paciente se debía a otro germen; sin embargo, los resultados negativos de los 27 cultivos realizados alejan esta posibilidad, dejando al Paracoccidioides como el agente causal del shock séptico.

Por otro lado, la presencia de hipercalcemia se ha visto relacionada con el diagnóstico de paracoccidioidomicosis en algunos reportes ${ }^{(7)}$. Aunque no se ha determinado con certeza su fisiopatología, parece ser que está relacionada con el incremento de conversión de hidroxivitamina $D$ a 1a,25dihidroxivitamina $D$ por parte de los macrófagos ${ }^{(8)}$, situación que también se ha observado en enfermedades granulomatosas como sarcoidosis y tuberculosis. Esta última fue descartada en nuestra paciente con los resultados negativos de 2 cultivos de Ziehl Neelsen y 1 estudio de quantiferon - TB gold plus. Cabe mencionar que se descartó hiperparatiroidismo, insuficiencia adrenal y no se reportaron lesiones osteolíticas en las múltiples radiografías de tórax.

Pese al tratamiento instaurado, la evolución de la paciente inicialmente fue desfavorable; probablemente por el desarrollo de un síndrome hemofagocítico evidenciado por anemia persistente, esplenomegalia, fiebre, hipertrigliceridemia, hiperferritinemia, y hemofagocitosis en el aspirado de médula ósea. Es importante mencionar que en nuestra institución no contamos con estudios de receptor alpha soluble de IL-2, ni test de degranulación/función de NK. Se decidió el uso de corticoides e inmunoglobulina humana IV a dosis de $2 \mathrm{gr}$ por $\mathrm{kg}$. Esta última requirió ser administrada una segunda vez por persistencia de los criterios clínicos y laboratoriales, luego de lo cual se observó mejoría clínica. Se descartó infección por los virus de Epstein-Barr, Herpes y Rubeola. A nuestro entender sólo se ha reportado un caso de síndrome hemofagocítico en el contexto de paracoccidioidomicosis ${ }^{(9)}$, por lo que consideramos relevante esta presentación clínica.

Finalmente, el diagnóstico pudo haberse sospechado tempranamente. La paciente procedía de una zona endémica ${ }^{(10)}$, presentaba clínica sugerente como fiebre, linfadenopatías, hepatoesplenomegalia, lesiones dérmicas, anemia, hipoalbuminemia, hipergammaglobulinemia e hipercalcemia; pero, fue con el AMO que se direccionó el tratamiento a la paracoccidioidomicosis. De ahí la enseñanza de este reporte, siempre considerar el antecedente 
epidemiológico, los detalles a la presentación de la enfermedad y realizar los estudios complementarios oportunamente.

\section{CONCLUSIÓN}

El shock séptico, la hipercalcemia y el síndrome hemofagocítico son complicaciones raras de la paracoccidioidomicosis. Sin embargo, el antecedente epidemiológico y los estudios complementarios como el AMO pueden orientar rápidamente el diagnóstico y tratamiento oportuno, que, de no brindarse tendría consecuencias fatales.

\section{REFERENCIAS BIBLIOGRÁFICAS}

1. Pereira RM, Bucaretchi F, Barison E de M, Hessel G, Tresoldi AT. Paracoccidioidomycosis in children: clinical presentation, follow-up and outcome. Rev Inst Med Trop São Paulo. 2004 Jun;46(3):127-31.

2. Restrepo A, McEWEN JG, Eda EC. The habitat of Paracoccidioides brasiliensis: how far from solving the riddle? Med Mycol. 2001 Jun;39(3):9.

3. Martinez R. New Trends in Paracoccidioidomycosis Epidemiology. J
Fungi. 2017 Jan 3;3(1):1.

4. Mendes RP, Cavalcante R de S, Marques SA, Marques MEA, Venturini J, Sylvestre TF, et al. Paracoccidioidomycosis: Current Perspectives from Brazil. Open Microbiol J. 2017 Oct 31;11(1):224-82.

5. Romaneli MT das N, Tardelli NR, Tresoldi AT, Morcillo AM, Pereira RM. Acute-subacute paracoccidioidomycosis: A paediatric cohort of 141 patients, exploring clinical characteristics, laboratorial analysis and developing a non-survival predictor. Mycoses. 2019 Nov;62(11):999-1005.

6. Shikanai-Yasuda MA, Telles Filho F de Q, Mendes RP, Colombo AL, Moretti ML. Consenso em paracoccidioidomicose. Rev Soc Bras Med Trop. 2006 Jun;39(3):297-310.

7. Almeida RM, Cezana L, Tsukumo DML, de Carvalho-Filho MA, Saad MJA. Hypercalcemia in a patient with disseminated paracoccidioidomycosis: a case report. J Med Case Reports. 2008 Dec;2(1):262.

8. Cadranel J, Garabedian M. $1,25(\mathrm{OH}) 2 \mathrm{D} 3$ Production by T Lymphocytes and Alveolar Macrophages Recovered by Lavage from Normocalcemic Patients with Tuberculosis. J Clin Invest. 1990 May;85(5):6.

9. Navarro AM, Pérez P, Arrunategui AM, Cañas CA. Paracoccidioidomicosis diseminada asociada a hipercalcemia y síndrome hemofagocítico. ACTAMÉDICA Colomb. 2008 Oct;33(4):4.

10. Zurita Macalupú S. Esporotricosis y paracoccidioidomicosis en Perú: experiencias en prevención y control. Rev Peru Med Exp Salud Pública [Internet]. 2014 Jul 2 [cited 2020 Oct 30];31(2). Available from: https://rpmesp.ins.gob.pe/index.php/rpmesp/article/view/58. 